
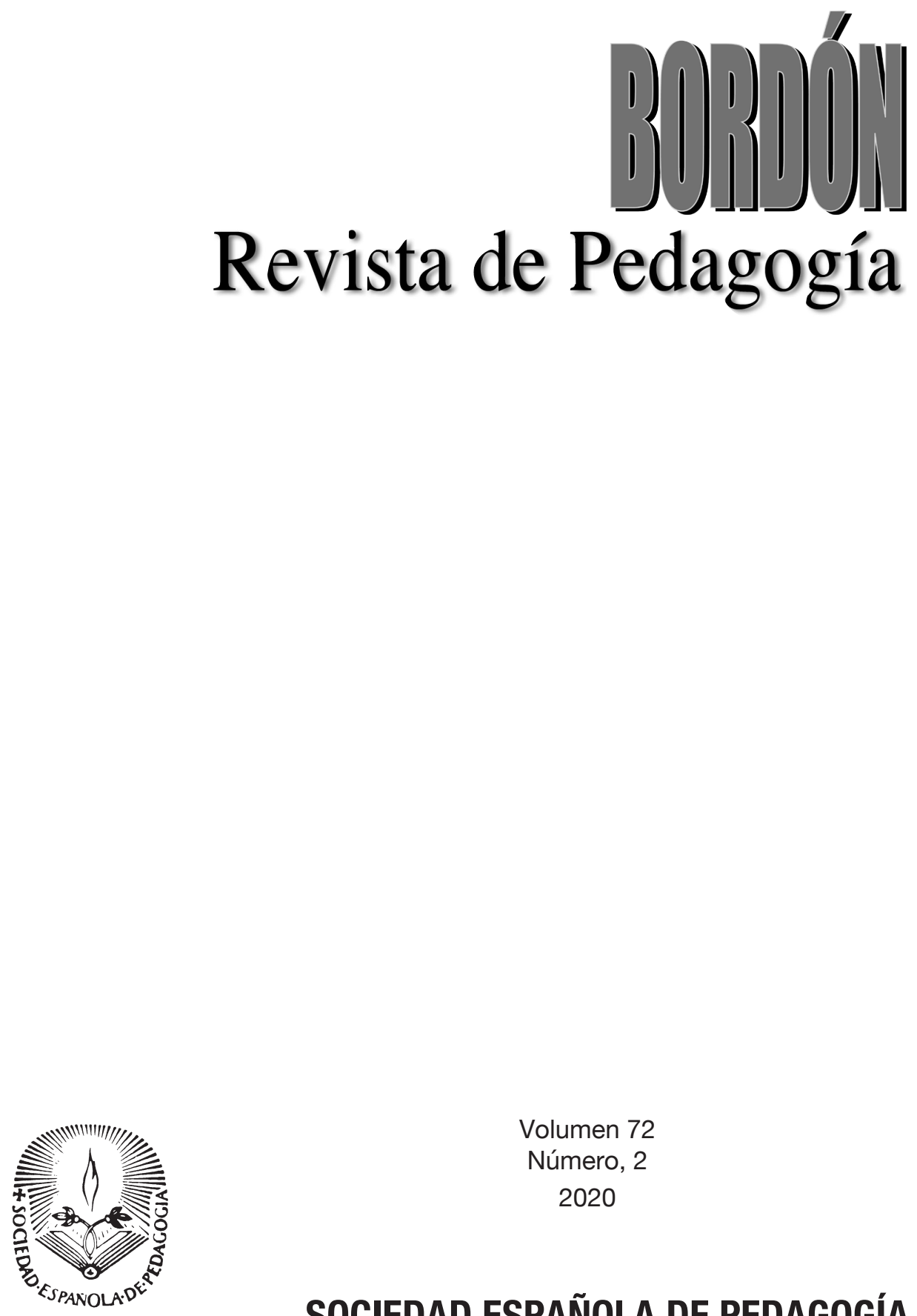

Volumen 72

Número, 2

2020 


\title{
COMPETENCIA DE EMPRENDIMIEnTO En EDUCACIÓn SECUNDARIA: PERCEPCIÓn DEL PROFESORADO SOBRE EL ESTADO ACTUAL Y LAS POSIBILIDADES FUTURAS EN EL CONTEXTO EUROPEO
}

\section{Education for entrepreneurship in Secondary Education: teachers' opinions about the current state and future possibilities in European contexts}

\author{
M. ${ }^{a}$ PAZ PRENDES ESPINOSA(1), ISABEL M. ${ }^{a}$ SOLANO FERNÁNDEZ ${ }^{(1)}$, JUAN GONZÁLEZ MARTÍNEZ(2) \\ Y FERNANDO CERDÁN CARTAGENA(3) \\ (I) Universidad de Murcia (España) \\ (2) Universitat de Girona (España) \\ (3) Universidad Politécnica de Cartagena (España)
}

DOI: 10.13042/Bordon.2020.67626

Fecha de recepción: 25/09/2018 - Fecha de aceptación: 27/10/2019

Autora de contacto / Corresponding author: M. ${ }^{2}$ Paz Prendes Espinosa. E-mail: pazprend@um.es

INTRODUCCIÓN. La educación para el emprendimiento se presenta como uno de los objetivos prioritarios de los sistemas educativos de los Estados miembros de la Unión Europea. Apoyando su definición y caracterización en el concepto de competencia de emprendimiento, en este artículo presentamos las capacidades vinculadas al desarrollo de esta competencia clave a partir de la propuesta hecha por el Marco Europeo para la Competencia Emprendedora. MÉTODO. Esta investigación se ha realizado desde un enfoque metodológico de carácter cuantitativo, basado en el cuestionario como instrumento de recogida de información. La muestra ha quedado conformada por 272 profesores de educación secundaria de Grecia, España, Italia y Bélgica. RESULTADOS. Las capacidades de emprendimiento más importantes para el profesorado son la motivación, el aprendizaje a partir de la experiencia y el trabajo en equipo; al desarrollo de estas capacidades es a lo que más tiempo dedican en clase, y asimismo, a lo que ellos creen que deben dedicar más tiempo. Además, los docentes demandan más formación para incluir la educación para el emprendimiento en su práctica docente; y, para ello, piden especialmente modelos y referentes prácticos que les ayuden a definir su acción docente. También se halla una baja inclusión de las tecnologías digitales como favorecedores del emprendimiento en el aula. DISCUSIÓN. La visión de los profesores coincide con la revisión de la literatura, si bien se detecta una diferencia significativa entre la importancia que los modelos teóricos asignan a la tecnología educativa y la importancia que le otorgan los profesores. Sin embargo, demandan ser formados al respecto, tanto en los referentes tecnológicos como en los pedagógicos.

Palabras clave: Emprendimiento, Competencia, Educación secundaria, Formación, Profesorado. 


\section{Introducción: la formación por competencias}

Esta investigación ha sido llevada a cabo en el marco del proyecto MOTIVA, financiado por la Unión Europea (véase http://motiva-project.eu), en el cual hemos estudiado cómo los profesores de secundaria contemplan la necesidad de trabajar la competencia de emprendimiento en las aulas y en el marco de la enseñanza reglada en tanto que competencia transversal básica. En el proyecto han participado instituciones de España, Italia, Grecia y Bélgica. En este artículo recogemos el estudio cuantitativo realizado para conocer el estado del arte sobre la competencia de emprendimiento en la enseñanza secundaria en opinión de los propios profesores. Hemos utilizado un cuestionario ad hoc basado en el modelo europeo de la competencia de emprendimiento y nuestros resultados apoyan la necesidad de trabajarla en la etapa de secundaria, pues en algunos casos es aquella en la que los estudiantes dan por concluidos sus estudios.

El tema central de nuestra investigación, por tanto, es la enseñanza por competencias. Como sabemos, las competencias han adquirido un significativo valor en el contexto de la pedagogía en las últimas décadas, tanto en el aspecto teórico y de investigación como en el ámbito de la práctica educativa real. El origen del concepto que actualmente manejamos lo encontramos en el psicólogo David McClelland (1973), experto en la teoría de la motivación, que planteó por primera vez la necesidad de evaluar por competencias, y con ello impuso un nuevo modo de gestión empresarial, que posteriormente sería reproducido en otros ámbitos, entre ellos, el educativo (Marina, 2010).

Hay muchas definiciones de competencia (Le Boterf, 2000; Cano, 2008; Gutiérrez, 2011; Marín, 2017; Monereo, 2005; Pavié, 2011; Perrenoud, 2004). De todas ellas nos quedamos con el concepto de Pavié (2011: 77): "La competencia es un grupo de elementos combinados (conocimientos, destrezas, habilidades y capacidades) que se movilizan e integran en virtud de una serie de atributos personales, en contextos concretos de acción". Por su parte, Cano (2008) señala tres elementos de las competencias: 1) articulan el conocimiento conceptual, procedimental y actitudinal en relación con nuestra capacidad para aplicarlo a la resolución de problemas; 2) se vinculan a rasgos de personalidad, pero también se aprenden y se pierden incluso; y 3) la acción les da sentido (se transfiere el conocimiento a una situación práctica), pero no es una acción mecánica, sino que ha de ser fruto de la reflexión.

En el marco del proyecto DeSeCo (OCDE, 2005) y, posteriormente, la Comisión Europea (2006) y Kampylis, Punie y Devine (2015) definen y clasifican las competencias clave para el ciudadano del siglo XXI, entre las cuales encontramos la competencia para el emprendimiento.

La competencia de emprendimiento es definida por la Comisión Europea (2006: 11) como la capacidad de innovación, creatividad y asunción de riesgos, junto con "la habilidad para planificar y gestionar proyectos con el fin de alcanzar objetivos". Y se añade: "en esta competencia se apoyan todas las personas, no sólo en la vida cotidiana, en casa y en la sociedad, sino también en el lugar de trabajo [...] y es el cimiento de otras capacidades y conocimientos más específicos que precisan las personas que establecen o contribuyen a una actividad social o comercial". El emprendimiento ha estado vinculado desde su consideración en la normativa europea a la competencia de autonomía e iniciativa personal (OCDE, 2005), así como a la ciudadanía y responsabilidad cívica (Jones y Irelade, 2010). Autores como Mesquita, Lopes y Bredis (2016) insisten en la importancia de la formación en todas las dimensiones de la competencia, pues una sociedad innovadora y que quiera progresar ha de apoyarse en personas emprendedoras.

Por su parte, el informe GEM (2015) muestra datos desalentadores con relación al emprendimiento en España, pues está por debajo de la media europea y en sentido descendente en los 
últimos años. Algo más de un 40\% de los sujetos encuestados en este informe reconocen haber recibido formación específica para desarrollar su capacidad de emprendimiento. Estos datos corroboran la necesidad puesta de manifiesto por la Comisión Europea (2012) de revisar nuestros sistemas educativos para que se invierta esfuerzo en el desarrollo de competencias y habilidades que contribuyan a mejorar nuestra calidad de vida y nuestro bienestar social, competencias que, como hemos visto, incluyen el emprendimiento.

Numerosos trabajos en estos últimos años focalizan su atención en la formación de la competencia de emprendimiento (Arranz et al., 2017; Contreras-Velásquez et al., 2017; Shaidullina et al., 2018) y la educación para el emprendimiento (Bernal y Cárdenas, 2017; Jones y Irelade, 2010; Testa y Francheri, 2015). Aunque la formación para el emprendimiento se ha trabajado más en el nivel de enseñanza superior, es igualmente cierto que cada vez se está acercando más esta formación a la enseñanza primaria y secundaria, como pone de manifiesto la experiencia realizada por BarbaSánchez y Atienza-Sahuquillo (2016).

Por todo lo visto, queda patente que el emprendimiento forma parte del catálogo de competencias clave del ciudadano del siglo XXI y además se recoge como imprescindible para el desarrollo de la formación permanente o formación a lo largo de la vida. Es por ello importante que la competencia de emprendimiento se trabaje desde el sistema de enseñanza formal. Es igualmente esencial entender que esta competencia está relacionada con capacidades que enriquecen la formación integral de la ciudadanía y no solamente hay que enfocarla desde el punto de vista de creación de empresas.

\section{La educación para el emprendimiento en el contexto europeo}

La educación para el emprendimiento se presenta como uno de los objetivos prioritarios de los Estados miembros de la Unión Europea. De hecho, es una materia cubierta por la educación escolar (primaria, secundaria y formación profesional) en todos los países de la red Eurydice, a excepción de Alemania, Irlanda y Liechtenstein (Comisión Europea/EACEA/Eurydice, 2016).

La mayoría de los países de la UE toman como base las competencias clave formuladas en el proyecto DeSeCo (OCDE, 2005) y la Comisión Europea (2006) para definir la educación para el emprendimiento. En su caracterización es preciso incidir en la necesidad de no restringir la educación emprendedora al ámbito del conocimiento de la empresa y los negocios (Bernal y Cardenas, 2014; Jones y Irelade, 2010; Marina, 2010; Testa y Francheri, 2015). Como indica el informe Eurydice de 2016, la educación para el emprendimiento no se reduce solo al contexto de la actividad laboral y empresarial, sino que se completa con la vida de la persona (Comisión Europea/EACEA/Eurydice, 2016). En este sentido, hablaremos de espíritu potencial emprendedor entendido "desde la perspectiva educacional, como el cúmulo de capacidades que un sujeto puede desarrollar configurando su identidad emprendedora" (Bernal y Cárdenas, 2017: 75). Y, en esta misma línea, Wibawo, Saptono y Suparno (2018) señalan la necesidad de construir enfoques interdisciplinares, no centrados exclusivamente en una formación para crear empresas, de modo que la educación para el emprendimiento sea accesible a todos los estudiantes.

Las capacidades que contribuirán al desarrollo del espíritu emprendedor han sido propuestas por diversos autores. Lumpkin y Dess (1996), desde un enfoque más centrado en la orientación empresarial, identifica la autonomía, la innovación, la tolerancia al riesgo y la proactividad y competitividad como las capacidades básicas para el desarrollo del espíritu emprendedor. Por su parte, Athayde (2009), en una visión más actual apunta a la creatividad, el control personal, la orientación al logro, la resolución de problemas y el liderazgo como capacidades fundamentales. Por último, Bernal 
y Cárdena (2014) destacan las capacidades de autonomía, iniciativa, creatividad y espíritu cooperativo. Recientemente, el Marco Europeo para la Competencia Emprendedora (EntreComp) ha identificado tres grandes áreas y 15 subcompetencias distribuidas en torno a ellas (Bacigalupo, Kampylis, Punie y Van den Brande, 2016):

a) Área de ideas y oportunidades. Incluye las subcompetencias de identificar oportunidades, creatividad, visión, evaluar ideas y pensamiento ético sostenible.

b) Área de recursos. Se refiere a recursos personales traducidos en las subcompetencias de autoconocimiento y confianza en sí mismo, motivación y perseverancia, movilización de recursos, educación financiera y económica e involucrar a otras personas.

c) Área de pasar a la acción. Abarca las subcompetencias de tomar la iniciativa, planificar y gestionar, manejar la incertidumbre, la ambigüedad y el riesgo, aprender de la experiencia y trabajar con otras personas.

Bernal y Cardenas (2017) realizan un análisis de programas de educación emprendedora en el contexto español y los resultados ponen de manifiesto que su efecto en el potencial emprendedor ha sido irrelevante, debido a la falta de formación específica del profesorado, las deficiencias en el diseño de programas, la falta de implicación para la implementación de programas de emprendimiento, la carencia de contenidos ajustados a esta temática y una evaluación educativa incorrecta. En este sentido, son algunos los autores que apuestan porque la educación para el emprendimiento se enseñe desde un enfoque metodológico basado en el aprendizaje basado en la acción (Jones y Irelade, 2010; Testa y Frascheri, 2015). Ruskovaara y Pihkala (2015) señalan además el aprendizaje cooperativo, el aprendizaje en equipo, el trabajo por proyectos y el aprendizaje basado en juegos.
El papel otorgado al profesorado en el exitoso desarrollo de la educación para el emprendimiento parece fundamental (Bernal y Cárdenas, 2017); por ello, la Comisión Europea (2006) apunta que los profesores deberían recibir una formación individualizada y específica; y Testa y Frascheri (2015) añaden que además deberían disponer de recursos para planificar, aplicar y evaluar la educación para el emprendimiento. Más recientemente, Purwana, Suhud, Fatimah y Armelita (2018) concluyen en una investigación realizada con estudiantes de secundaria que era necesario que los docentes fueran más innovadores en el uso de métodos de aprendizaje, reclamando así una mejora en la formación del profesorado de este nivel educativo.

De una u otra forma, existen evidencias de que tanto en España como en el resto de los países de la Unión Europea, la educación para el emprendimiento no se ha integrado plenamente o con éxito en los contextos educativos (Bernal y Cárdenas, 2017; Europea/EACEA/Eurydice, 2016). Por ello, se hace necesario analizar la perspectiva del profesorado, sus concepciones y sus acciones, para formarlo y contribuir con ello al desarrollo de la educación para el emprendimiento. Y, por ello, adquiere interés el estudio que hemos realizado a partir de la concreción de nuestras preguntas de investigación: ¿consideran los profesores de secundaria que es importante abordar la formación de la competencia de emprendimiento desde la enseñanza reglada?, ¿están abordando ya de alguna manera esta competencia? Y si es así, ¿qué aspectos de la competencia de emprendimiento se están trabajando en las aulas de secundaria?

\section{Metodología}

Esta investigación se enmarca en el proyecto MOTIVA, concedido al amparo de la Unión Europea en el marco de la convocatoria Erasmus+ 2016. El propósito de este proyecto es potenciar las competencias del alumnado de educación secundaria (entre 12 y 18 años) a través del uso 
de una plataforma en la nube diseñada en el marco del proyecto.

Para el desarrollo de esta investigación se optó por un enfoque metodológico de carácter cuantitativo, basado en el cuestionario como instrumento de investigación. Siguiendo una estrategia descriptiva, en los términos expuestos por Ato, López y Benavente (2013), planteamos una investigación no experimental, de tipo exploratorio. Para ello, se determinaron a priori, tras la revisión de la literatura, las capacidades que podrían servir para desarrollar la competencia de emprendimiento en el alumnado de educación secundaria, tomando como base el Marco Europeo de Competencias de Emprendimiento (EntreComp) (Bacigalupo, Kampylis, Punie y Van den Brande, 2016). Estas capacidades fueron analizadas a partir de las actividades didácticas llevadas a cabo por los docentes, su opinión y sus necesidades formativas, poniéndolas en relación entre sí. Por ello, consideramos que se trata de una investigación con un diseño descriptivo-correlacional, ya que de acuerdo con lo expuesto por Hernández, Fernández y Baptista (2010) para este tipo de estudios hemos considerado pertinente establecer la relación teórica entre las capacidades seleccionadas para este estudio, que se enumeran en la tabla 1, y la relación existente entre ellas, o con otras variables como el género.

\section{Instrumento}

El cuestionario elaborado ad hoc para esta investigación ${ }^{1}$ consta de 114 ítems agrupados en torno a 41 preguntas, de las que 4 (7 ítems) son cuestiones de tipo sociodemográfico. Tras las preguntas sociodemográficas, el cuestionario se organiza en dos partes: en la primera, formada por 81 ítems, se pide la valoración del profesorado sobre las capacidades que permitirán el desarrollo de las competencias de emprendimiento; y la segunda (26 ítems) se centra en las características de una plataforma ideal. Esta segunda parte del instrumento no ha sido incluida en este artículo, pues ha sido analizada

TABLA 1. Capacidades de emprendimiento consideradas para la elaboración del instrumento

\begin{tabular}{|c|c|}
\hline Capacidades & Indicadores \\
\hline Creatividad & Ser curioso y abierto, desarrollar ideas, definir problemas, diseñar valor, ser innovador \\
\hline Trabajar con otros & $\begin{array}{l}\text { Aceptar la diversidad (las diferencias de la gente), desarrollar la inteligencia } \\
\text { emocional, escuchar activamente, formar equipo, trabajar juntos, expandir tu red }\end{array}$ \\
\hline Usar dispositivos móviles & $\begin{array}{l}\text { Inspirar e inspirarse, persuadir, comunicarse eficazmente, usar los medios de } \\
\text { comunicación con eficacia }\end{array}$ \\
\hline Autoconfianza & $\begin{array}{l}\text { Perseguir sus aspiraciones, identificar fortalezas y debilidades, creer en su capacidad, } \\
\text { moldear su futuro }\end{array}$ \\
\hline $\begin{array}{l}\text { Enfrentarse con } \\
\text { la incertidumbre, la } \\
\text { ambigüedad y el riesgo }\end{array}$ & Hacer frente a la incertidumbre y la ambigüedad, calcular el riesgo, gestionar el riesgo \\
\hline $\begin{array}{l}\text { Aprender a través de } \\
\text { la experiencia }\end{array}$ & Reflexionar, aprender a aprender, aprender de la experiencia. \\
\hline $\begin{array}{l}\text { Planificar y gestionar } \\
\text { proyectos }\end{array}$ & $\begin{array}{l}\text { Definir objetivos, planificar y organizar, definir prioridades, monitorizar su progreso, } \\
\text { ser flexible y adaptarse a los cambios }\end{array}$ \\
\hline Motivación y perseverancia & $\begin{array}{l}\text { Ser constante, tener determinación, centrarse en lo que te mantiene motivado, ser } \\
\text { resistente, no rendirse }\end{array}$ \\
\hline Movilización de otros & $\begin{array}{l}\text { Inspirar e inspirarse, persuadir, comunicarse eficazmente, usar los medios de } \\
\text { comunicación con eficacia }\end{array}$ \\
\hline
\end{tabular}


de forma independiente y se relaciona con otros objetivos de la investigación no directamente ligados al análisis de la competencia de emprendimiento, sino al análisis de plataformas telemáticas para trabajar con estudiantes de secundaria. En la tabla 1 se enumeran las capacidades analizadas en el cuestionario, así como una breve descripción.

Todos los ítems responden a una escala de carácter ordinal de 5 valores (tipo Likert de acuerdodesacuerdo), y de frecuencia que permiten un análisis descriptivo y correlacional de los datos. Asimismo, se trata de un tipo de escala ampliamente usada en investigación educativa, como pone de manifiesto la revisión sistemática realizada recientemente por Matas (2018). Del mismo modo, decidimos usar una escala de 5 valores, con una opción de indiferente, asumiendo los argumentos datos por autores como Schuman y Presser (1981), entre otros. En este primer bloque referido a la competencia de emprendimiento, fue validado mediante juicio de expertos de diferentes ramas de conocimiento y países. El instrumento mostró una fiabilidad general muy alta, alpha $=.927$. Para precisar aún más la confiabilidad del instrumento, hemos incluido en la tabla 2 el valor alpha de Cronbach obtenido por los ítems incluidos en cada una de las capacidades analizadas. Es preciso indicar además que en el cálculo de descriptivos para escala si se elimina algún elemento de la lista, la correlación elemento-total corregida de cada ítem por cada una de las capacidades analizadas ha sido en todos los casos inferior a 0,30, encontrándose las correlaciones entre valores mínimos de 0,391 y máximos de 0,774 , por lo que no se justifica la eliminación de ningún ítem de la escala para analizar la percepción del profesorado sobre las capacidades de emprendimiento.

\section{Participantes}

Para la realización de este estudio exploratorio, se distribuyó el cuestionario en línea a docentes de educación secundaria de cuatro países de la Unión Europea: Bélgica, Grecia, Italia y España, elegidos por constituir los países de los miembros participantes en el proyecto. La muestra productora de datos quedó conformada por 272 informantes, un 39,7\% de Bélgica, un 20,2\% de Grecia, un $17,6 \%$ de Italia y un 22,4\% de España (gráfico 1). La técnica de muestreo usada fue no probabilística por conveniencia. Si bien es cierto que la gran limitación de este tipo de muestreo es la nula posibilidad de generalización de los resultados (Etikan, Abubakar y

Tabla 2. Coeficiente alpha de Cronbach para las capacidades analizadas

Capacidades

\begin{tabular}{ll} 
Creatividad & 0,761 \\
\hline Trabajar con otros & 0,755 \\
\hline Usar dispositivos móviles & 0,895 \\
\hline Autoconfianza & 0,808 \\
\hline Enfrentarse a incertidumbre, la ambigüedad y el riesgo & 0,820 \\
\hline Aprender a través de la experiencia & 0,850 \\
\hline Planificar y gestionar proyectos & 0,861 \\
\hline Motivación y perseverancia & 0,846 \\
\hline Movilización de otros & 0,870
\end{tabular}


Gráfico 1. País de procedencia de los informantes (valores de \% redondeados)

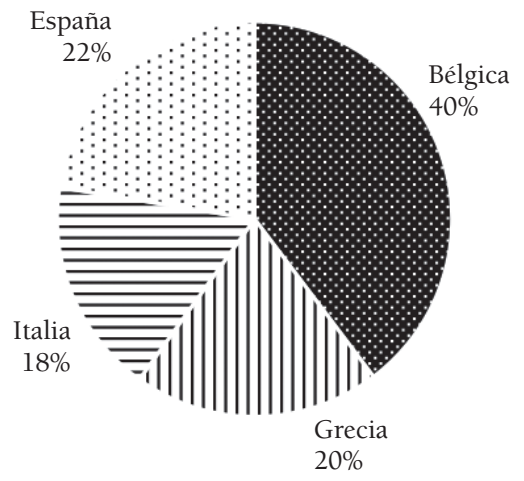

Sunusi), es preciso indicar que esta no es una de las aspiraciones de la investigación, ya que tiene un carácter exploratorio. Por tanto, para la selección de la muestra le dimos prioridad a criterios como los indicados por Dörnyei (2007), como la facilidad de acceso a la muestra, la voluntad de participar en el estudio o la proximidad geográfica respecto a los colaboradores en la investigación.

Un $64,7 \%$ eran mujeres y un $35,3 \%$ hombres. Y en cuanto a los biodatos que tienen que ver con su ejercicio profesional, un 33,8\% dedicaban la mayor parte de su jornada al primer ciclo de la educación secundaria (12-13 años), un 34,6\% al ciclo medio de la secundaria (14-15 años) y el restante 31,6 se centraban en el ciclo superior de la secundaria y el bachillerato (16-17 años). Finalmente, también era diversa su experiencia profesional docente, si bien el grupo de profesores noveles (con menos de 5 años de experiencia) era el menos numeroso (gráfico 2).

\section{Procedimiento y análisis de datos}

El cuestionario se administró en línea a través de la plataforma de formularios de Google Drive. El análisis estadístico se efectuó con los paquetes IBM SPSS v.21. Los estadísticos se estimaron con el 95\% de fiabilidad. Se realizó un análisis descriptivo y otro exploratorio (diagramas de tallo

\section{Gráfico 2. Años de experiencia profesional docente del profesorado}

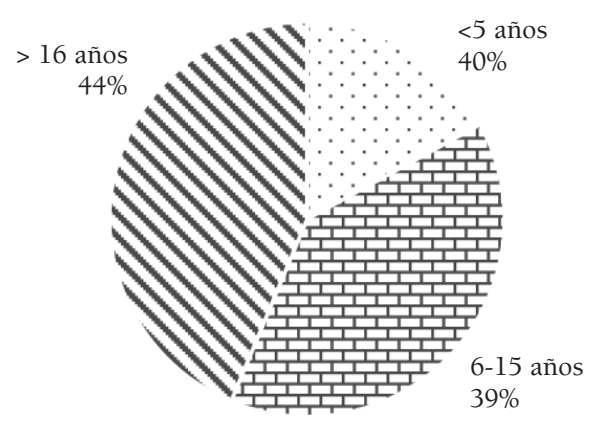

y hoja), junto con un análisis cruzado por medio de tablas de contingencia. En la medida en que las pruebas de normalidad no aseguraron una distribución normal de la muestra, se usó una prueba de chi-cuadrado para las pruebas de significación de la diferencia.

\section{Resultados}

Atendiendo al instrumento elaborado para llevar a cabo este estudio, recogemos a continuación los resultados referidos a la primera parte del cuestionario, es decir, la valoración que el profesorado de la muestra realiza sobre las capacidades que permitirán el desarrollo de las competencias de emprendimiento.

A partir de aquí, puede analizarse la importancia que los informantes otorgan a cada una de las capacidades de emprendimiento. En primer lugar, los elementos más importantes que el profesorado considera fomentar en el alumnado de secundaria son la motivación $(4,77)$, el aprendizaje a partir de la experiencia propia $(4,74)$ y el trabajo en equipo $(4,72)$, lo que por otro lado entronca con algunas de las preocupaciones generales del profesorado de esta etapa educativa. Curiosamente, en aquel ítem que obtiene la menor valoración, el aprendizaje de las tecnologías móviles al servicio del emprendimiento, la disparidad de valores es la mayor, 
como se refleja en la desviación estándar de este ítem, superior a las demás. Así pues, como vemos en la tabla 3, en una escala de Likert de 1 a 5 , los valores medios y las desviaciones estándares fueron los siguientes:

TABLA 3. Importancia asignada a las capacidades de emprendimiento (escala de 1 a 5 )

\begin{tabular}{lcc}
\hline & Media & Desv. est. \\
\hline Creatividad & 4,45 & 0,805 \\
\hline Trabajo en equipo & 4,72 & 0,615 \\
\hline Uso de tecnologías móviles & 3,81 & 1,081 \\
\hline Autoevaluación & 4,52 & 0,703 \\
\hline Gestión de la incertidumbre & 4,48 & 0,672 \\
\hline Experiencia & 4,74 & 0,529 \\
\hline Planificación & 4,62 & 0,699 \\
\hline Motivación & 4,77 & 0,495 \\
\hline Liderazgo & 4,06 & 0,905
\end{tabular}

A continuación, podemos ver ahora cuál es la disponibilidad horaria que asignarían para el desarrollo de cada una de estas capacidades. Los docentes son más favorables a dedicar parte de su dedicación docente a capacidades como el trabajo en equipo, la motivación, la creatividad o el aprendizaje a partir de la propia experiencia, que a otras como el liderazgo, el uso de las tecnologías móviles o la planificación de los propios aprendizajes (véase que, en estos tres últimos casos, hay una parte importante de los informantes que no está dispuesto a dedicarles ningún tiempo en sus sesiones; y, en oposición, las capacidades que hemos indicado al inicio son aquellas en las que más porcentaje de profesores se considera dispuesto a dedicarles más de 8 horas de la docencia presencial) (gráfico 3).

Por otro lado, y respecto a esta disponibilidad horaria que los docentes declaran, en el gráfico 4 encontramos la dedicación real media que el profesorado reconoce que ha impartido en los dos años últimos a cada una de estas capacidades, en una escala de 1 (nada) a 5 (siempre, continuamente). En cierta forma, podemos ver que seguimos el patrón que se intuía al hablar de la disponibilidad del profesorado para integrar las diferentes capacidades. Y así, aquellas que menos se han trabajado en el aula son el liderazgo y las tecnologías móviles al servicio del aprendizaje; y, en oposición, el trabajo en equipo, la motivación, la creatividad y el aprendizaje a partir de la experiencia son aquellas que más dedicación han recibido por parte del profesorado en la docencia presencial.

\section{GráfICO 3. Disponibilidad horaria por capacidades (en \%)}

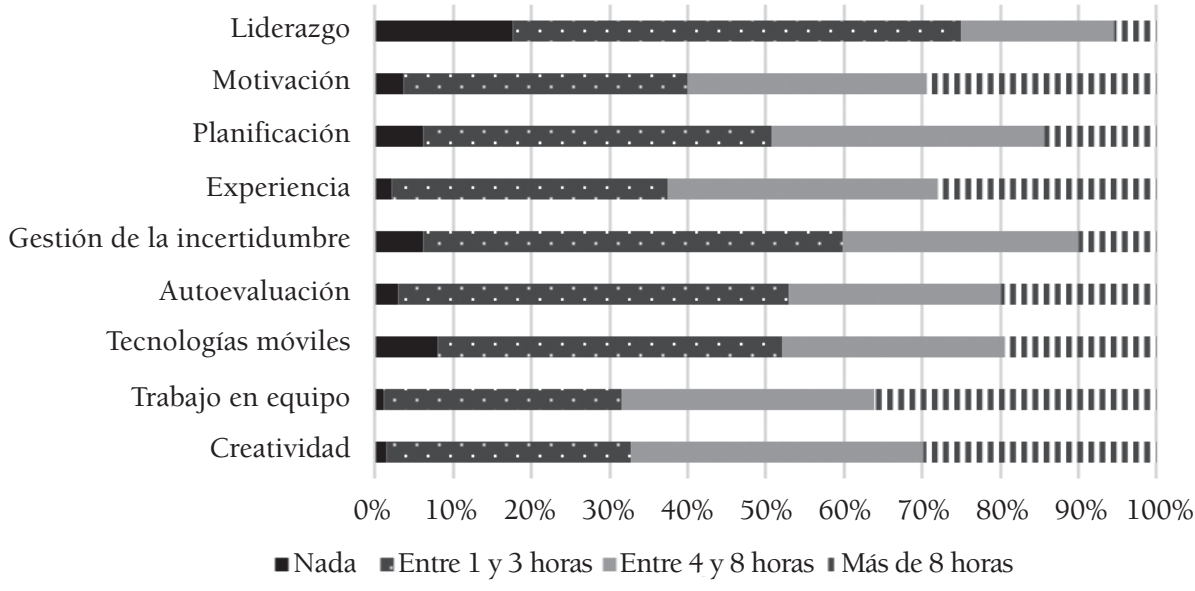


Gráfico 4. Dedicación media de los dos últimos años por capacidades (escala de 1 a 5 )

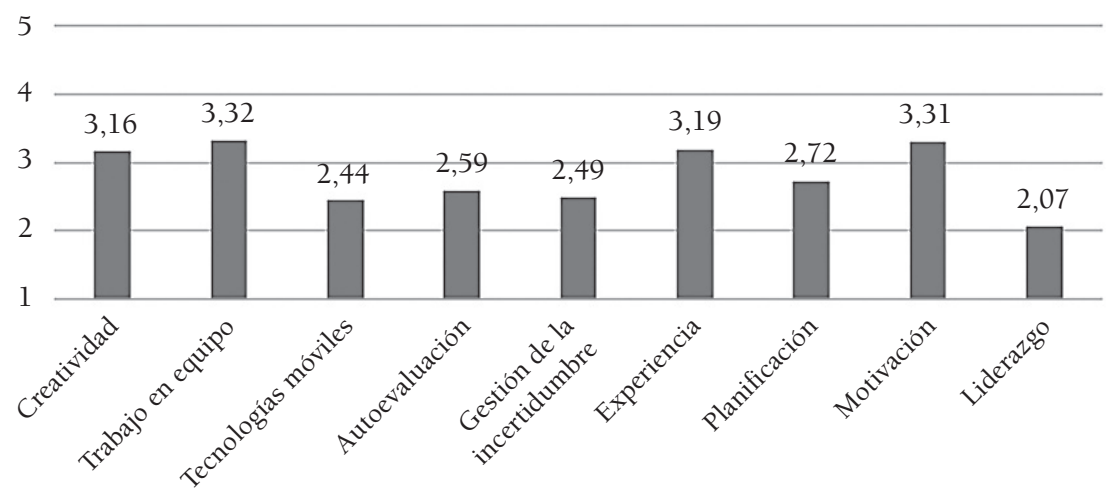

TABla 4. Correlaciones entre la importancia, la disponibilidad y la dedicación real a cada una de las capacidades (correlaciones de Spearman)

\begin{tabular}{lccc} 
& $\begin{array}{c}\text { Importancia y } \\
\text { disponibilidad }\end{array}$ & $\begin{array}{c}\text { Importancia y } \\
\text { dedicación }\end{array}$ & $\begin{array}{c}\text { Disponibilidad } \\
\text { y dedicación }\end{array}$ \\
\hline Creatividad & & & 0,471 \\
\hline Trabajo en equipo & 0,264 & 0,350 & 0,515 \\
\hline Uso de tecnologías móviles & 0,256 & 0,300 & 0,572 \\
\hline Autoevaluación & 0,430 & 0,425 & 0,511 \\
\hline Gestión de la incertidumbre & 0,294 & 0,346 & 0,586 \\
\hline Experiencia & 0,396 & 0,346 & 0,611 \\
\hline Planificación & 0,417 & 0,286 & 0,623 \\
\hline Motivación & 0,362 & 0,302 & 0,584 \\
\hline Liderazgo & 0,327 & 0,346 & 0,700
\end{tabular}

A partir de estas tres apreciaciones, podemos ver cuáles son las relaciones entre la importancia que consideran para cada capacidad, el tiempo que podrían dedicarle y el que le han dedicado en el pasado cercano. Y, en efecto, vemos en la tabla 4 que las relaciones que se establecen entre la importancia, la dedicación y la disponibilidad de cada una de las capacidades son todas significativas $(<0,01)$, de modo que podemos asumir que la relación entre todas las variables y la importancia, la dedicación y la disponibilidad es directa (se valora más la creatividad, planificación o la motivación, por ejemplo, cuanto mayores son las tres variables de referencia que se reflejan en las columnas).
No obstante, es destacable que estas correlaciones, aunque significativas, no son especialmente intensas. Así, si atendemos a las correlaciones entre la importancia que asignan a cada capacidad y la disponibilidad horaria que consideran para esa misma capacidad; y lo mismo ocurre entre la importancia de cada capacidad y la dedicación real que ellos mismos indican. Sin embargo, son más intensas entre la dedicación real y la disponibilidad horaria; esto, a priori, parece indicarnos que el profesorado está más predispuesto a dedicar más tiempo a aquello que ya viene ocupándole que a aquello que considera importante, de manera general, con independencia de la capacidad de que se trate. 
A partir de esta primera descripción general, podemos adentrarnos en el análisis de la diferencia en función de las variables independientes tenidas en cuenta en el estudio, para ver si estas primeras consideraciones son generales o existen variaciones significativas. Para ello, y en la medida en que los tests de parametricidad nos indican que la distribución de la muestra no cumple las condiciones de normalidad, acudimos a las correspondientes pruebas de chicuadrado, que nos muestran los indicadores de significación que se recogen en la tabla 5. Para facilitar la lectura, sombreamos aquellos que están por debajo del nivel de confianza de 0,05. Como vemos, la variable de país y la de género (por ese orden) son las más productivas; y, por el contrario, la experiencia profesional docente y el nivel educativo en el que este cubre la mayor parte de su jornada se muestran menos interesantes desde el punto de vista descriptivo.

En cuanto al país de procedencia, como se puede ver en la tabla 5, los datos ponen de manifiesto que el profesorado belga concede una importancia considerablemente menor a las capacidades de creatividad, autoevaluación, gestión de la incertidumbre y aprendizaje a través de la experiencia (gráfico 5 y tabla 6). Por su parte, los docentes griegos son los que menor importancia conceden al aprendizaje a través de las tecnologías móviles. Y, en cuanto a los españoles, son los que más importante consideran la

Tabla 5. Significación de la diferencia en función del género, el país de procedencia, el nivel preferente de dedicación docente o la experiencia docente (nivel de significación del 0,05)

\begin{tabular}{|c|c|c|c|c|}
\hline & Género & País & Nivel & Experiencia \\
\hline Creatividad & 0,220 & 0,000 & 0,071 & 0,682 \\
\hline Trabajo en equipo & 0,032 & 0,425 & 0,301 & 0,671 \\
\hline Uso de tecnologías móviles & 0,008 & 0,000 & 0,093 & 0,022 \\
\hline Autoevaluación & 0,004 & 0,009 & 0,920 & 0,192 \\
\hline Gestión de la incertidumbre & 0,371 & 0,018 & 0,205 & 0,931 \\
\hline Experiencia & 0,235 & 0,008 & 0,352 & 0,846 \\
\hline Planificación & 0,631 & 0,089 & 0,541 & 0,694 \\
\hline Motivación & 0,384 & 0,069 & 0,789 & 0,179 \\
\hline Liderazgo & 0,052 & 0,000 & 0,899 & 0,192 \\
\hline
\end{tabular}

Tabla 6. Medias y varianzas de las percepciones de importancia de las capacidades de emprendimiento (escala de 1 a 5)

\begin{tabular}{|c|c|c|c|c|c|c|c|c|c|c|}
\hline & \multicolumn{2}{|c|}{ Bélgica } & \multicolumn{2}{|c|}{ Grecia } & \multicolumn{2}{|c|}{ Italia } & \multicolumn{2}{|c|}{ España } & \multicolumn{2}{|c|}{ Total } \\
\hline & Media & $s^{2}$ & Media & $s^{2}$ & Media & $s^{2}$ & Media & $s^{2}$ & Media & $s^{2}$ \\
\hline Creatividad & 4,14 & 0,850 & 4,69 & 0,218 & 4,73 & 0,202 & 4,55 & 0,760 & 4,45 & 0,648 \\
\hline Tec. móviles & 3,96 & 0,858 & 3,6 & 1,059 & 3,21 & 1,360 & 4,18 & 1,217 & 3,81 & 1,169 \\
\hline Autoevaluación & 4,32 & 0,576 & 4,76 & 0,258 & 4,67 & 0,270 & 4,55 & 0,625 & 4,52 & 0,495 \\
\hline Incertidumbre & 4,35 & 0,539 & 4,44 & 0,436 & 4,53 & 0,341 & 4,72 & 0,342 & 4,48 & 0,451 \\
\hline Experiencia & 4,65 & 0,305 & 4,74 & 0,271 & 4,83 & 0,227 & 4,85 & 0,265 & 4,74 & 0,280 \\
\hline Liderazgo & 3,86 & 0,794 & 4,02 & 1,018 & 4,08 & 0,418 & 4,43 & 0,826 & 4,06 & 0,819 \\
\hline
\end{tabular}


Gráfico 5. Diferencias por países en la percepción de importancia de las capacidades de emprendimiento (escala de 1 a 5 )

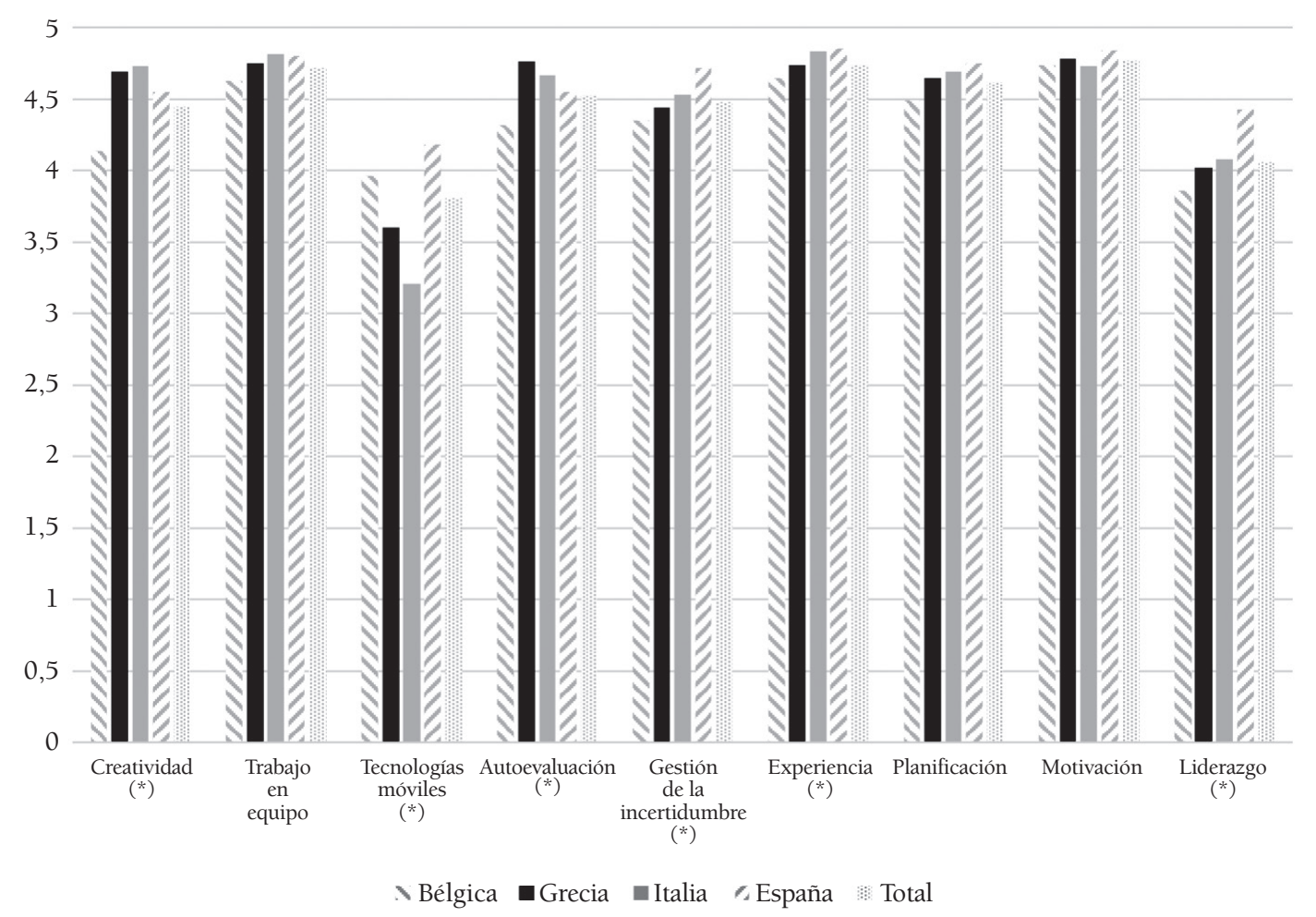

capacidad de liderazgo, aprendizaje a partir de la experiencia y de la gestión de la incertidumbre y por medio de las tecnologías móviles.

En relación con el género también vemos aspectos interesantes. En cuanto a la importancia asignada a las diferentes capacidades, observamos que las profesoras asignan de modo general mayor importancia que sus compañeros a las capacidades de trabajo en equipo, uso de las tecnologías móviles y autoevaluación de los aprendizajes. Como en el caso anterior, pueden verse los valores $p$ de significación en la tabla 4 .

Finalmente, si atendemos a las capacidades en las que la diferencia es significativa por intervalos de experiencia profesional docente, vemos en la tabla 7 que el único punto en que la variable de experiencia docente se muestra relevante tiene que ver con la importancia que se asigna al uso de las tecnologías móviles al servicio del aprendizaje. En concreto, vemos que son los docentes con menor experiencia profesional aquellos que menos importante consideran esta categoría, en contraste con los docentes con una experiencia media, que son quienes la otorgan una mayor importancia.

Por último, para acabar ya con este epígrafe de resultados, podemos atender a lo que el profesorado entiende que son sus necesidades de formación para incorporar efectivamente el emprendimiento en la docencia secundaria. En este sentido, en la tabla 8 encontramos el grado de acuerdo de los docentes con la importancia de formarse en cada uno de los ítems que se les proponía. Y, a partir de las medias de cada uno de estos ítems, como podemos apreciar, sus 
Gráfico 6. Diferencias por género en la percepción de la importancia de las capacidades de emprendimiento (escala de 1 a 5)

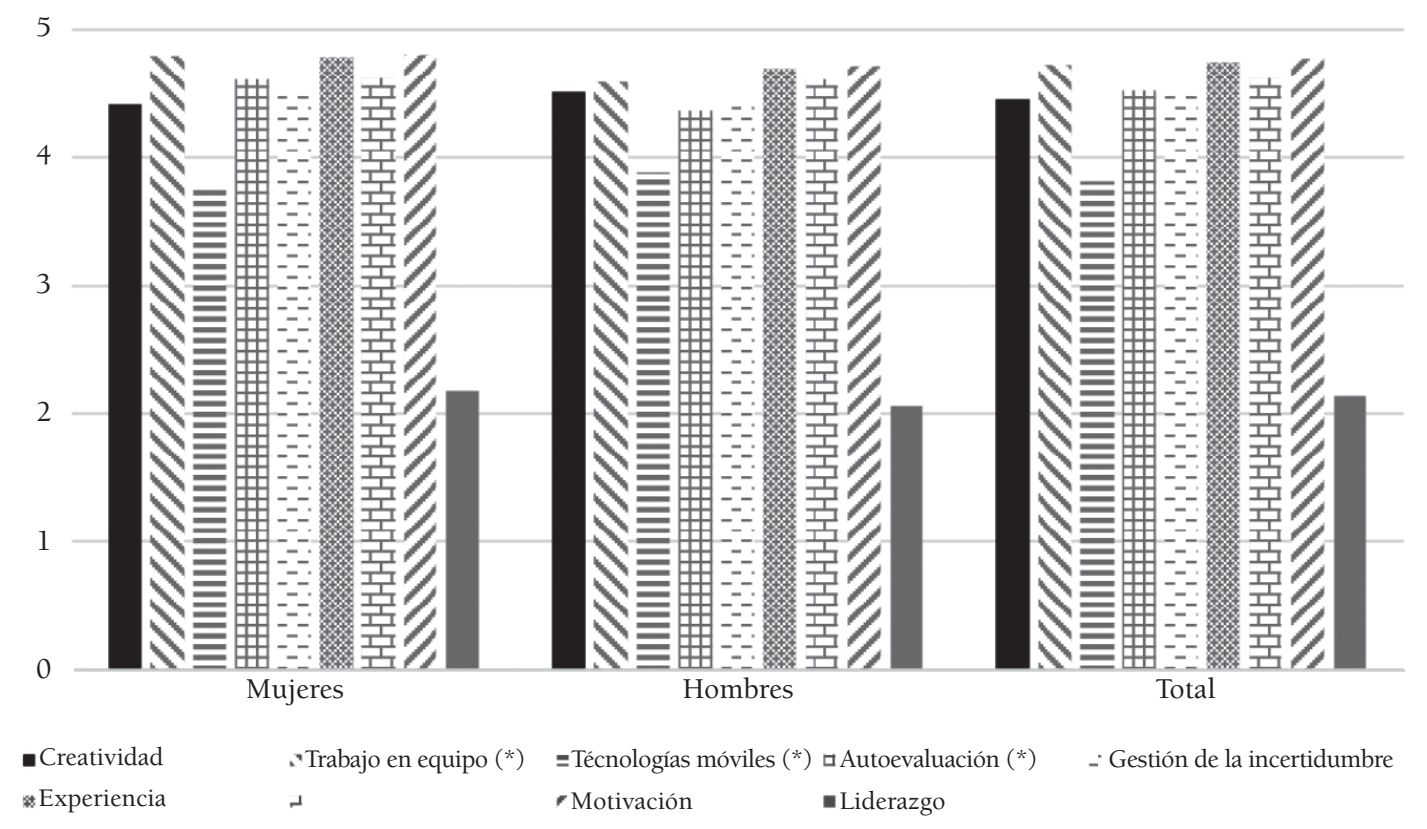

TABla 7. Diferencias por experiencia profesional docente en la percepción de la importancia de las capacidades de emprendimiento (escala de 1 a 5 )

$<5$ años

Tecnologías móviles
6-15 años

3,67
3,95
$>16$ años

3,73

Total

TABla 8. Necesidades de formación del profesorado para la docencia de emprendimiento (escala de 1 a 5)

\begin{tabular}{lcc} 
& Media & StD \\
\hline Guías didácticas & 3,98 & 0,800 \\
\hline Buenas prácticas & 4,31 & 0,577 \\
\hline Plataformas & 3,94 & 0,737 \\
\hline Grupos interdisciplinares & 3,94 & 0,792 \\
\hline Recursos móviles & 3,86 & 0,805 \\
\hline Evaluación & 4,27 & 0,700
\end{tabular}

principales demandas tienen que ver con disponer de buenas prácticas que les sirvan de modelo para diseñar situaciones de aprendizaje y con mejorar las estrategias de evaluación de los aprendizajes. En segundo lugar, formarse en la elaboración de guías didácticas o en la gestión de grupos interdisciplinares con los que trabajar en estos diseños, y los dos aspectos de índole más tecnológica, la utilización de plataformas de aprendizaje y de recursos móviles al servicio del aprendizaje. 


\section{Discusión y conclusiones}

Tras este análisis, queda justificada la importancia de trabajar el emprendimiento en el contexto europeo. Ningún profesor europeo pondría en cuestión el desarrollo de esta competencia clave. Aun así, una realidad constatada son las carencias en la formación del profesorado, que se revela como una de las piezas clave para el desarrollo exitoso de la educación para el emprendimiento (Bernal y Cárdenas, 2017; Comisión Europea, 2006; Ruskovaara y Pihkala, 2013 y 2015; Testa y Frascheri, 2015; Purwana et al., 2018). En este sentido, el informe Eurydice pone de manifiesto que "es necesario realizar avances particulares en dos áreas: los resultados de aprendizaje y la formación del profesorado" (Comisión Europea/EACEA/Eurydice, 2016: 18). Asimismo, Ruskovarra y Pihkala apuntan que los resultados muestran la importancia de la formación del profesorado en la introducción de nuevas perspectivas pedagógicas en la escuela, entre las que se encuentra la educación para el emprendimiento. Atendiendo a las evidencias de los resultados de las investigaciones realizadas sobre este tema, estaríamos de acuerdo en afirmar que la selección del profesorado debe ser cuidadosa, ya que ellos cumplen un papel fundamental en la eficacia de los planes de la educación emprendedora (Seikkula-Leino, Ruskovaara, Ikävalko, Mattila y Rytkölä, 2010).

Asumiendo esta premisa, se ha ahondado en conocer la perspectiva y la dedicación del profesorado de secundaria a las capacidades para el desarrollo de la educación emprendedora. De las nueve capacidades analizadas, extraídas a partir de la propuesta del Marco Europeo EntreComp, las más destacadas han sido la motivación, la creatividad, el trabajo en equipo y el aprendizaje a partir de la experiencia. Estas capacidades no solo son las consideradas más importantes por el profesorado, sino que también son a las que más tiempo dedica el profesorado europeo que ha servido de base para realizar este estudio, y a su vez, a las que más tiempo están dispuestos a dedicar en su futura práctica docente. En nuestro estudio, se pone de manifiesto que existe una relación significativa entre la importancia, la dedicación real y la disponibilidad futura de dedicación de las capacidades analizadas. Asimismo, los datos reflejan que el profesorado está más predispuesto a dedicar más tiempo a aquello de lo que ya viene ocupándose que a lo que considera más importante, lo cual quizá tiene que ver con una tradicional resistencia al cambio de la población docente (Blase, 2005; Strebel, 2006; Starr, 2011). Este aspecto será una de las líneas de investigación en las que podremos ahondar en futuras investigaciones.

Por otro lado, una de las capacidades menos valoradas y trabajadas en el aula por el profesorado de educación secundaria es el aprendizaje con dispositivos móviles. Este dato parece ir en la contra de la repercusión actual que las tecnologías digitales están teniendo en el contexto empresarial y, en particular, en las competencias emprendedoras. Nambisan (2016) examina el impacto de la digitalización en el ámbito empresarial y valora la repercusión que este puede tener en la necesidad de realizar una nueva teorización del emprendimiento. Por su parte, Giones y Brem (2017) señalan que la revolución digital ha contribuido a la transformación de los significados y la formación del espíritu emprendedor en todo el mundo. Realizan una distinción sólida y relevante entre el emprendimiento tecnológico, el emprendimiento tecnológico digital y el emprendimiento digital, cuya consideración en los planes de estudio de la educación para el emprendimiento de los Estados miembros de la Unión Europea podría replantear la importancia que el profesorado de esta etapa educativa otorga al aprendizaje con dispositivos móviles. En este sentido, un dato alentador en nuestro estudio se obtiene de la evidencia del reconocimiento por parte del profesorado de la necesidad manifiesta de formación en el uso de plataformas tecnológicas y de recursos móviles al servicio del aprendizaje. 
Este estudio ha puesto de relieve la existencia de diferencias estadísticamente significativas en la percepción del profesorado europeo por países en relación con la importancia de las capacidades a trabajar en la educación para el emprendimiento. En este sentido, concluimos que el profesorado belga otorga más importancia a la creatividad, la autoevaluación, la gestión de la incertidumbre y el aprendizaje a partir de la experiencia. Por el contrario, el profesorado griego es el que menos importancia da al aprendizaje de dispositivos móviles y los españoles son los que valoran más las capacidades de liderazgo y el aprendizaje con dispositivos móviles. Las diferencias por género ponen de manifiesto que las profesoras otorgan más importancia a las capacidades de trabajo en equipo, el uso de dispositivos móviles y la autoevaluación de los aprendizajes. Por último, en relación con la experiencia docente, solo se han encontrado diferencias significativas respecto a la importancia otorgada al aprendizaje de los dispositivos móviles, y son los profesores con menos años de experiencia los que menos importancia dan a esta capacidad; mientras que aquellos que tienen una experiencia media son los que consideran que tiene más importancia.

Además de lo anterior, en este análisis no podríamos pasar por alto las necesidades formativas detectadas por el profesorado de educación secundaria en relación con el desarrollo de la competencia de emprendimiento en el alumnado de esta etapa educativa. La necesidad más reclamada es la referida a la necesidad de disponer de un catálogo de buenas prácticas de educación para el emprendimiento. Recordemos que, aunque ha quedado patente el fracaso de los programas de educación emprendedora en diferentes países europeos (Bernal y Cárdenas, 2017; Ruskovaara y Pihkala, 2015), la Comisión Europea (2014) está realizando un esfuerzo por recoger buenos ejemplos de educación emprendedora que pueden servir de guía al profesorado. Aun así, los resultados de nuestro estudio vienen a apoyar la propuesta de Ruskovaara y Pihkala (2015) que afirma que una manera eficaz de promover las políticas de educación para el emprendimiento sería introducir modelos de buenas prácticas y conceder poderosos incentivos para que el profesorado participe activamente en actividades escolares que redunden en la consecuencia de la competencia para el emprendimiento. En la misma línea, Purwana et al. (2018) apuntan la necesidad de implicación de los responsables de formular políticas educativas para la creación de un plan de estudios que incremente la motivación de emprendimiento de los alumnos de secundaria.

Para terminar, nuestro estudio concluye que el profesorado de educación secundaria necesita más formación en la elaboración de guías didácticas y gestión de grupos interdisciplinares. Las carencias de formación del profesorado de esta etapa se derivan del modelo consecutivo de formación del profesorado de educación secundaria generalizado en Europa (Bolívar, 2007; Lorenzo, Muñoz y Beas, 2015; Rebolledo, 2015). Siguiendo a Bolívar (2007: 24), en una clara apuesta por el modelo concurrente que actualmente se desarrolla en la formación del profesorado inicial de infantil y primaria, el modelo consecutivo "nunca podrá dar lugar a una identidad profesional de partida, sino — si acasopor recomposiciones o reconstrucciones". Se desprende así de este resultado, la necesidad de reflexionar sobre la mejora de la formación didáctica del profesorado de educación secundaria y de introducir cambios políticos que contribuyan al desarrollo de prácticas educativas exitosas en esta etapa educativa.

\section{Limitaciones y líneas futuras}

Es necesario reconocer que, aunque los datos aportados nos ofrecen una primera aproximación de gran interés al tema, el estudio presenta limitaciones respecto a la muestra, pues no incluye a todos los países de Europa o de una zona geográfica concreta. Sería necesario, en este sentido, abordar en futuros estudios un análisis más amplio, basado en el contraste de 
hipótesis, con datos que nos permitiesen comparar la realidad en todos y cada uno de los países europeos. O incluso se podría afrontar un estudio a más amplia escala en cada país estudiado, pues el instrumento ha demostrado su fiabilidad y validez para medir el objeto de estudio y responder a las preguntas de investigación.

Además de ello, el propio instrumento incluía un segundo bloque relativo al análisis de plataformas telemáticas que puedan ser útiles para la enseñanza secundaria. Es un estudio que podrá aportar luz sobre el uso de tecnologías en esta etapa de la enseñanza y que igualmente supone una de las líneas de trabajo a abordar en el futuro.
Consideramos que las conclusiones obtenidas pueden ser de interés para los agentes educativos y también para los responsables políticos, pues aportan luz sobre la acción educativa en relación con la competencia de emprendimiento que, a pesar de la relevancia dada por el Marco Europeo ya citado, creemos que no es objeto de suficiente atención en los centros de secundaria. Y en relación con ello, abordar iniciativas de formación del profesorado para que aprendan a implementar estrategias específicas que trabajen todas las capacidades de esta competencia, tanto en la formación inicial (el máster de secundaria) como en las iniciativas de formación permanente.

\section{Nota}

${ }^{1}$ El cuestionario puede ser consultado en http://bit.ly/2XUgrAo (consultado el 30 de octubre de 2019).

\section{Referencias bibliográficas}

Athayde, R. (2009). Measuring potential in young people. Entrepreneurship Theory and Practice, 33(2), 481-500. doi: http://doi.org/10.1111/j.1540-6520.2009.00300.x

Ato, M., López, J. J. y Benavente, A. (2013). Un sistema de clasificación de los diseños de investigación en psicología. Anales de Psicología, 29(3), 1038-1059. doi: http://doi.org/10.6018/analesps.29.3.178511

Arranz, N., Ubierna, F, Arroyabe, M. F., Pérez, C. y Fernández de Arroyabe, J. C. (2017). The effect of curricular and extracurricular activities on university students' entrepreneurial intention and competences. Studies in Higher Education, 42(11,2), 1979-2008. doi: http://doi.org/10.1080/030 75079.2015.1130030

Bacigalupo, M., Kampylis, P. Punie, Y. y Van den Brande, G. (2016). EntreComp: The Entrepreneurship Competence Framework. Joint Research Centre. doi: http://doi.org/10.2791/593884

Barba-Sánchez, V. y Atienza-Sahuquillo, S. (2016). The development of entrepreneurship at school: the Spanish experience. Education + Training, 58, 1-16. doi: http://doi.org/10.1108/ET-01-20160021

Bernal-Guerrero, A. y Cárdenas-Gutiérrez, A. R. (2014). La formación de emprendedores en la escuela y su repercusión en el ámbito personal. Una investigación narrativa centrada en el programa EME. Revista Española de Pedagogía, 257, 125-144. Recuperado de https://reunir.unir.net/ handle/123456789/3752

Bernal-Guerrero, A. y Cárdenas-Gutiérrez, A. R. (2017). Evaluación del potencial emprendedor en escolares. Una investigación longitudinal. Educación XX1, 20(2), 73-94. doi: http://doi. org/10.5944/edu- cXX1.14162

Blase, J. (2005). The micropolitics of educational change. En A. Hargreaves (ed.), Extending educational change: international handbook of educational change (pp. 264-277). Dordrect: Springer. 
Bolívar, A. (2007). La formación inicial del profesorado de secundaria y su identidad profesional. Revista Estudios sobre Educación, 12, 13-30. doi: http://doi.org/10.15581/004.12.13-30

Cano García, M. E. (2008). La evaluación por competencias en la educación superior. Profesorado. Revista de Currículum y Formación de Profesorado, 12(3), 1-16. Recuperado de https://www.ugr. es/ recfpro/rev123COL1.pdf

Comisión Europea (2006). Competencias clave para el aprendizaje permanente. Recomendación 2006/962/CE del Parlamento Europeo y del Consejo, de 18 de diciembre de 2006, sobre las competencias clave para el aprendizaje permanente [Diario Oficial L 394 de 30.12.2006] Recuperado de http: /eur-l ex.europa.eu/LexUriSer v/site/es/oj/2006/1_394/1_39420061230es00100018.pdf

Comisión Europea (2012). Rethinking education: investing in skills for better socio-economic outcomes. Recuperado de http://ec.europa.eu/education/news/rethinking/com669_en.pdf

Comisión Europea (2014). Entrepreneurship education. A guide for educators. doi: http://doi. org/10.2769/51003.

Contreras-Velásquez, J. C., Wilches-Durán, S. Y., Graterol-Rivas, M. E. y Bautista-Sandoval, M. J. (2017). Educación superior y la formación en emprendimiento interdisciplinario: un caso de estudio. Formación Universitaria, 10(3), 11-20. doi: http://doi.org/10.4067/S071850062017000300003

Dörnyei, Z. (2007). Research methods in applied linguistics. Nueva York: Oxford University Press. doi: http://doi.org/10.5054/tj.2010.215611

Etikan, I., Abubakar, S. y Sunusi, A. (2015). comparison of convenience sampling and purposive sampling. American Journal of Theoretical and Applied Statistics, 5(1), 1-4. doi: http://doi. org/10.11648/j.ajtas.20160501.11

European Commission/EACEA/Eurydice (2016). La educación para el emprendimiento en los centros educativos en Europa. Informe de Eurydice. Luxemburgo: Oficina de Publicaciones de la Unión Europea.

GEM (2015). Global entrepreneurship monitor. Informe GEM España 2014. Recuperado de http://www. cise.es/wp-content/uploads/Informe-GEM-Espa\%C3\%B1

Giones, F. y Brem, A. (2017). Digital technology entrepreneurship: a definition and research agenda. Technology Innovation Management Review, 7(5), 44-51. Recuperado de http://timreview.ca/ article/1076

Gutiérrez Porlán, I. (2011). Competencias del profesorado universitario en relación al uso de tecnologías de la información y comunicación: Análisis de la situación en España y propuesta de un modelo de formación. Tesis doctoral. Universidad Rovira i Virgili, Tarragona. Recuperado de http://hdl. handle.net/10803/52835

Hernández, R., Fernández, C. y Baptista, P. (2010). Metodología de la investigación. México: McGraw-Hill. Jones, B. e Irelade, N. (2010). Enterprise education as pedagogy. Education and Training, 52(1), 7-19. doi: http://doi.org/10.1108/00400911011017654.

Kampylis, P., Punie, Y. y Devine, J. (2015). Promoting effective digital-age learning - A European framework for digitally-competent educational organisations. doi: http://doi.org/10.2791/54070

Le Boterf, G. (2000). La ingeniería de las competencias. Barcelona: Gestión 2000.

LOMCE (2013). Ley Orgánica 8/2013, de 9 de diciembre, para la mejora de la calidad educativa. Recuperado de https://www.boe.es/buscar/pdf/2013/BOE-A-2013-12886-consolidado.pdf

Lorenzo, J. A., Muñoz, I. M. y Beas, M. (2015). Modelos de formación inicial del profesorado de Educación Secundaria en España desde una perspectiva europea. Revista Complutense de Educación, 26(3), 741-757. doi: http://doi.org/10.5209/revRCED.2015.v26.n3.44866.

Lumpkin, G. T. y Dess, G. G. (1996). Clarifying the entrepreneurial orientation construct and linking it to performance. The Academy of Management Review, 21(1), 135-172. Recuperado de http://www.jstor.org/stable/258632 
Marín Trejo, R. (2017). Diseño y evaluación de un instrumento de la competencia digital docente. Tesis doctoral. Universidad de Islas Baleares.

Marina, J. A. (2010). La competencia de emprender. Revista de Educación, 351, 49-71. Recuperado de http://www.revistaeducacion.educacion.es/re351/re351_03.pdf

Matas, A. (2018). Diseño del formato de escalas tipo Likert: un estado de la cuestión. Revista Electrónica de Investigación Educativa, 20(1), 38-47. doi: http://doi.org/10.24320/redie.2018.20.1.1347

McClelland, D. (1973). Testing for competence rather than for "intelligence". American Psichologist, 28, 1-14. Recuperado de https://www.therapiebreve.be/documents/mcclelland-1973.pdf

Mesquita, C., Lopes, R. P. y Bredis, K. (2016). Entrepreneurship in Higher Education as a horizontal competence. En M. Peris-Ortiz, J. Alonso, F. Vélez-Torres y C. Rueda-Armengot (eds.), Education tools for entrepreneurship. Creating an action-learning environment through educational learning tools (pp. 223-246). doi: http://doi.org/10.1007/978-3-319-24657-4

Monereo, C. (2005). Internet y competencias básicas. Barcelona: Graó.

Nambisan, S. (2016). Digital entrepreneurship: toward a digital technology perspective of entrepreneurship. Entrepreneurship Theory and Practice, 414, 1-27. doi: http://doi.org/10.1111/etap.12254

OCDE (2005). The definition and selection of key competences. Executive summary. Recuperado de http:// www.oecd.org/pisa/35070367.pdf. http://deseco.ch/bfs/deseco/en/index/03/02.parsys.78532.downloadList.94248.DownloadFile.tmp/2005.dscexecutivesummary.sp.pdf

Pavié, A. (2011). Formación docente: hacia una definición del concepto de competencia profesional docente. Revista Electrónica Interuniversitaria de Formación del Profesorado, 14(1), 67-80. Recuperado de http//www.aufop.com

Perrenoud, P. (2004). Diez nuevas competencias para enseñar. Barcelona: Graó.

Purwana, D., Suhud, U., Fatimah, T. y Armelita, A. (2018). Antededents of secondary students entrepreneurial motivation. Journal of Entrepreneurship Education, 21(2), 1-7.

Real Decreto 126/2014, de 28 de febrero, por el que se establece el currículo básico de la Educación Primaria. Recuperado de https://www.boe.es/buscar/pdf/2014/BOE-A-2014-2222-consolidado.pdf.

Real Decreto 1105/2014, de 26 de diciembre, por el que se establece el currículo básico de la Educación Secundaria Obligatoria y del Bachillerato. https://www.boe.es/boe/dias/2015/01/03/pdfs/ BOE-A-2015-37.pdf

Rebolledo, T. (2015). La formación inicial del profesorado de Educación Primaria y Secundaria en Alemania, España, Finlandia, Francia y Reino Unido. Estudio Comparado. Revista Española de Educación Comparada, 25, 129-148. doi: http://doi.org/10.5944/reec.25.2015.14787

Ruskovaara, E. y Pihkala, T. (2013). Teachers implementing entrepreneurship education: classroom practices. Education and Training, 55 (2), 204-216. doi: http://doi.org/10.1108/00400911311304832

Ruskovaara, E. y Pihkala, T. (2015). Entrepreneurship education in schools: empirical evidence on the teacher's role. The Journal of Educational Research, 108, 236-249. doi: http://doi.org/10.1080/ 00220671.2013 .878301

Seikkula-Leino, J., Ruskovaara, E., Ikävalko, M., Mattila, J. y Rytkölä, T. (2010). Promoting entrepreneurship education: The role of the teacher? Education and Training, 52(2), 117-127. doi: http://doi.org/10.1108/00400911011027716.

Schuman, H. y Presser, S. (1981). Questions and answers in attitude surveys. San Diego, CA: Academic Press.

Shaidullina, A. R., Zakirova, V. G., Kashurnikov, S. N., Arestova, E. N., Shmidt, A. N. y Kovaleva, N. I. (2018). Students training for innovative entrepreneurial activity: social responsibility competences. Espacios, 39(2). Recuperado de http://www.revistaespacios.com/a18v39n02/a18v39n02p15.pdf

Starr, K. (2011). Principals and the politics of resistance to change. Educational Management Administration and Leadership, 39(6), 646-660. 
Strebel, P (2006). Why do employees resist change? Harvard Business Review on Change. Boston, MA: Harvard Business School Press.

Testa, S. y Francheri, S. (2015). Learning by failing: What we can learn from un-successful entrepreneurship education. The International Journal of Management Education, 13(1), 11-22. doi: http:// doi.org/10.1016/j.ijme.2014.11.001

Wibowo, A., Saptono, A. y Suparno, N. J. (2018). Does teacher' creativity impact on vocational students' entrepreneurial intention? Journal of Entrepreneurship Education, 21(2), 1-7.

\begin{abstract}
Education for entrepreneurship in Secondary Education: teachers' opinions about the current state and future possibilities in European contexts
\end{abstract}

INTRODUCTION. Education for entrepreneurship is considered one of the priority goals for the educational systems by the EU member states. Supporting the definition and the characterization of the concept of entrepreneurship competence, in this paper we present the capacities linked to the development of this key competence, taking as a starting point the proposal made by the European Entrepreneurship Competence Framework (EntreComp). METHOD. This research has been carried out using a quantitative methodological approach, based on the questionnaire as an instrument for collecting information. The sample has been made up of 272 Secondary Education teachers from Greece, Spain, Italy and Belgium. RESULTS. The most important elements for teachers are motivation, learning from experience and teamwork. They are also those elements with more presence in the classroom, and those which teachers think they should devote more time to in class. In addition, teachers demand more training to include education for entrepreneurship in their teaching practice; and, for this, they especially require models and practical references that help them to define their teaching action. There is also a low inclusion of digital technologies as favouring entrepreneurship in the classroom. DISCUSSION. The teachers' views are in line with the literature review, although an important difference is detected between the importance that the theoretical models have and the importance teachers assign to educational technology. However, they demand to be trained this way as much in the technological referents as in the pedagogical ones.

Keywords: Entrepreneurship, Competence, Secondary Education, Teacher, Training.

\title{
Résumé
}

Compétence entrepreneuriale dans l'enseignement secondaire: état actuel et possibilités futures dans le contexte européen, d'après le point de vue des enseignants

INTRODUCTION. Les systèmes éducatifs des États membres de l'Union européenne ont plusieurs objectifs, dont l'enseignement de l'entrepreneuriat est présentée comme prioritaire. Après de réfléchir sur le concept de compétence entrepreneuriale, nous présentons dans cet article les capacités associées au développement de cette compétence clé dans le cadre européen pour la compétence en entrepreneuriat. MÉTHODE. Cette recherche a été réalisée à partir d'une approche méthodologique quantitative, basée sur un questionnaire comme outil de collecte des données. L'échantillon de 
recherche comprend 272 enseignants du secondaire de la Grèce, l'Espagne, l'Italie et la Belgique. RÉSULTATS. Les éléments les plus importants pour les enseignants sont la motivation, l'apprentissage par l'expérience et le travail d'équipe. Ceux-ci sont aussi les éléments auxquels les enseignants consacrent plus de temps en et auxquels ils pensent qu'il faut consacrer encore plus de temps dans la salle de classe. Les enseignants exigent également à cet égard de cours de formation pour introduire l'enseignement de l'entrepreneuriat dans leurs propres pratiques d'enseignement. Ils demandent notamment des modèles et des références pratiques qui leur aident à définir leurs stratégies pédagogiques. On observe également une faible utilisation des technologies numériques pour favoriser l'entrepreneuriat dans les salles de classe. DISCUSSION. Les conclusions des enseignants coïncident avec celles obtenues à partir de la révision de la littérature scientifique. Néanmoins, une différence significative entre l'importance que les modèles théoriques attribuent à la technologie éducative et l'importance que les enseignants lui accordent a été identifiée. Dans le cas particulier des enseignants, ils demandent une formation technologique aussi bien théorique que pédagogique.

Mots-clés : Entrepreneuriat, Compétence, Éducation Secondaire, Enseignant, Formation.

\section{Perfil profesional de los autores}

\section{M. ${ }^{\text {a }}$ Paz Prendes Espinosa (autora de contacto)}

Doctora en Ciencias de la Educación y catedrática de Didáctica y Organización Escolar en la Universidad de Murcia. Directora del Grupo de Investigación en Tecnología Educativa (GITE) de la Universidad de Murcia y directora de RIITE, Revista Interuniversitaria de Investigación en Tecnología Educativa. Secretaria de EDUTEC, Asociación para el Desarrollo de la Tecnología Educativa. Coordinadora en la Universidad de Murcia del Programa de Doctorado Interuniversitario en Tecnología Educativa. Especialista en el uso de tecnologías como herramientas educativas, e-learning y competencia digital. Actualmente directora del proyecto EmDigital sobre la competencia de emprendimiento digital en estudiantes universitarios y directora del proyecto europeo AROSE sobre el uso de tecnologías para la adquisición de competencias lingüísticas en secundaria.

Correo electrónico: pazprend@um.es

Web: www.pazprendes.es

ORCID: https://orcid.org/0000-0001-8375-5983

Dirección para correspondencia: Facultad de Educación (Campus de Espinardo), Universidad de Murcia, 30100 Murcia (España).

\section{Isabel M. Solano Fernández}

Doctora en Pedagogía por la Universidad de Murcia. Profesora titular de Tecnología Educativa y, actualmente, directora del Departamento de Didáctica y Organización Escolar de la citada Universidad. Miembro del Grupo de Investigación de Tecnología Educativa (GITE). Entre sus líneas de investigación destacan los recursos multimedia audiovisuales en contexto de enseñanza formal y no formal, la competencia digital del profesorado, las estrategias metodológicas en contextos de enseñanza formal y no formal, el emprendimiento digital como competencia en la educación superior y la metodología de la investigación en tecnología educativa.

Correo electrónico: imsolano@um.es

ORCID: https://orcid.org/0000-0003-3760-8899 


\section{Juan González Martínez}

Profesor agregado Serra Húnter del Departament de Pedagogia de la Universitat de Girona. Doctor en Tecnología Educativa por la Universitat Rovira i Virgili (Tarragona, España) y en Lengua Española por la Universidad Nacional de Educación a Distancia (Madrid, España). Miembro del grupo de investigación consolidado UdiGitalEdu y de los grupos de innovación docente Transmèdia i Educació i Narratives Digitals en Educació Social de la Universitat de Girona. Profesor visitante del programa de doctorado en Tecnología Educativa de la Universitat Rovira i Virgili y de la Universidad Autónoma de Querétaro (México). Sus principales intereses de investigación se centran en la competencia digital de los estudiantes y en la competencia digital docente, así como en el transmedia y su uso educativo.

Correo electrónico: juan.gonzalez@udg.edu

ORCID: https://orcid.org/0000-0002-9175-6369

\section{Fernando Cerdán Cartagena}

Catedrático de Ingeniería Telemática en la Universidad Politécnica de Cartagena y creador del grupo de investigación DINTEL (División de Innovación en Sistemas Telemáticos y Tecnología Electrónica). Dirige la cátedra INDRA-UPCT, donde desarrolla proyectos relacionados con las smart cities y el e-health. Resultado de su intensa labor en colaboración con empresas y como miembro del proyecto BAF (Broadband Access Facilities), obtuvo el premio al mejor proyecto europeo RACE. El producto «Plaisir», desarrollado en colaboración con la empresa Inforges, formó parte del catálogo de productos Linux de IBM. Es cofundador y CEO de IngeniaTIC Desarrollo, S. L, creadora, entre otros productos, del sistema CADI (Clase Docente Interactiva), de servicios de Telefonía IP sobre tecnología propia y de la conocida aplicación SmileYou. Cuenta con dos registros de propiedad intelectual, dos patentes con examen previo y un modelo de utilidad, todos en explotación comercial actualmente. Es el investigador principal del proyecto MOTIVA de la Comisión Europea.

Correo electrónico: fernando.cerdan@upct.es 